\title{
Fuzzy stability of a mixed type functional equation
}

Sun Sook Jin and Yang-Hi Lee*

* Correspondence: yanghi2@hanmail.net Department of Mathematics Education, Gongju National University of Education, Gongju 314-711, Republic of Korea

\section{Abstract}

In this paper, we investigate a fuzzy version of stability for the functional equation

$$
f(x+y+z)-f(x+y)-f(y+z)-f(x+z)+f(x)+f(y)+f(z)=0
$$

in the sense of Mirmostafaee and Moslehian.

1991 Mathematics Subject Classification. Primary 46S40; Secondary 39852.

Keywords: fuzzy normed space, fuzzy almost quadratic-additive mapping, mixed type functional equation

\section{Introduction}

A classical question in the theory of functional equations is "when is it true that a mapping, which approximately satisfies a functional equation, must be somehow close to an exact solution of the equation?". Such a problem, called a stability problem of the functional equation, was formulated by Ulam [1] in 1940. In the next year, Hyers [2] gave a partial solution of Ulam's problem for the case of approximate additive mappings. Subsequently, his result was generalized by Aoki [3] for additive mappings and by Rassias [4] for linear mappings, for considering the stability problem with unbounded Cauchy differences. During the last decades, the stability problems of functional equations have been extensively investigated by a number of mathematicians, see [5-17].

In 1984, Katsaras [18] defined a fuzzy norm on a linear space to construct a fuzzy structure on the space. Since then, some mathematicians have introduced several types of fuzzy norm in different points of view. In particular, Bag and Samanta [19], following Cheng and Mordeson [20], gave an idea of a fuzzy norm in such a manner that the corresponding fuzzy metric is of Kramosil and Michalek type [21]. In 2008, Mirmostafaee and Moslehian [22] obtained a fuzzy version of stability for the Cauchy functional equation:

$$
f(x+y)-f(x)-f(y)=0 .
$$

In the same year, they [23] proved a fuzzy version of stability for the quadratic functional equation:

$$
f(x+y)+f(x-y)-2 f(x)-2 f(y)=0 .
$$


We call a solution of (1.1) an additive map and a mapping satisfying (1.2) is called $a$ quadratic map. Now we consider the functional equation:

$$
f(x+y+z)-f(x+y)-f(y+z)-f(x+z)+f(x)+f(y)+f(z)=0 .
$$

which is called a mixed type functional equation. We say a solution of (1.3) a quadratic-additive mapping. In 2002, Jung [24] obtained a stability of the functional equation (1.3) by taking and composing an additive map $A$ and a quadratic map $Q$ to prove the existence of a quadratic-additive mapping $F$, which is close to the given mapping $f$. In his processing, $A$ is approximate to the odd part $\frac{f(x)-f(-x)}{2}$ of $f$ and $Q$ is close to the even part $\frac{f(x)+f(-x)}{2}$ of it, respectively.

In this paper, we get a general stability result of the mixed type functional equation (1.3) in the fuzzy normed linear space. To do it, we introduce a Cauchy sequence $J_{n} f$ $(x)\}$ starting from a given mapping $f$, which converges to the desired mapping $F$ in the fuzzy sense. As we mentioned before, in previous studies of stability problem of (1.3), they attempted to get stability theorems by handling the odd and even part of $f$, respectively. According to our proposal in this paper, we can take the desired approximate solution $F$ at once. Therefore, this idea is a refinement with respect to the simplicity of the proof.

\section{Fuzzy stability of the functional equation (1.3)}

We use the definition of a fuzzy normed space given in [19] to exhibit a reasonable fuzzy version of stability for the mixed type functional equation in the fuzzy normed linear space.

Definition 2.1. ([19]) Let $X$ be a real linear space. A function $N: X \times \mathbb{R} \rightarrow[0,1]$ (the so-called fuzzy subset) is said to be a fuzzy norm on $x$ if for all $x, y \in X$ and all $s$, $t \in \mathbb{R}$,

(N1) $N(x, c)=0$ for $c \leq 0$;

(N2) $x=0$ if and only if $N(x, c)=1$ for all $c>0$;

(N3) $N(c x, t)=N(x, t /|c|)$ if $c \neq 0$;

(N4) $N(x+y, s+t) \geq \min \{N(x, s), N(y, t)\}$;

(N5) $N(x, \cdot)$ is a non-decreasing function on $\mathbb{R}$ and $\lim _{t \rightarrow \infty} N(x, t)=1$.

The pair $(X, N)$ is called a fuzzy normed linear space. Let $(X, N)$ be a fuzzy normed linear space. Let $\left\{x_{n}\right\}$ be a sequence in $X$. Then, $\left\{x_{n}\right\}$ is said to be convergent if there exists $x \in X$ such that $\lim _{n \rightarrow \infty} N\left(x_{n}-x, t\right)=1$ for all $t>0$. In this case, $x$ is called the limit of the sequence $\left\{x_{n}\right\}$, and we denote it by $N-\lim _{n \rightarrow \infty} x_{n}=x$. A sequence $\left\{x_{n}\right\}$ in $X$ is called Cauchy if for each $\varepsilon>0$ and each $t>0$ there exists $n_{0}$ such that for all $n \geq n_{0}$ and all $p>0$ we have $N\left(x_{n+p}-x_{n}, t\right)>1-\varepsilon$. It is known that every convergent sequence in a fuzzy normed space is Cauchy. If each Cauchy sequence is convergent, then the fuzzy norm is said to be complete and the fuzzy normed space is called $a$ fuzzy Banach space.

Let $(X, N)$ be a fuzzy normed space and $\left(Y, N^{\prime}\right)$ a fuzzy Banach space. For a given mapping $f: X \rightarrow Y$, we use the abbreviation

$$
D f(x, y, z):=f(x+y+z)-f(x+y)-f(y+z)-f(x+z)+f(x)+f(y)+f(z)
$$

for all $x, y, z \in X$. For given $q>0$, the mapping $f$ is called a fuzzy q-almost quadraticadditive mapping, if 


$$
N^{\prime}(D f(x, y, z), s+t+u) \geq \min \left\{N\left(x, s^{q}\right), N\left(y, t^{q}\right), N\left(z, u^{q}\right)\right\}
$$

for all $x, y, z \in X$ and all $s, t, u \in(0, \infty)$. Now we get the general stability result in the fuzzy normed linear setting.

Theorem 2.2. Let $q$ be a positive real number with $q \neq \frac{1}{2}, 1$. And let $f$ be a fuzzy $q$ almost quadratic-additive mapping from a fuzzy normed space $(X, N)$ into a fuzzy Banach space $\left(Y, N^{\prime}\right)$. Then there is a unique quadratic-additive mapping $F: X \rightarrow Y$ such that for each $x \in X$ and $t>0$,

$$
N^{\prime}(F(x)-f(x), t) \geq \begin{cases}\sup _{t^{\prime}<t} N\left(x,\left(\frac{2-2^{p}}{3}\right)^{q} t^{\prime q}\right) & \text { if } 1<q, \\ \sup _{t^{\prime}<t} N\left(x,\left(\frac{\left(4-2^{p}\right)\left(2-2^{p}\right)}{6}\right)^{q} t^{\prime q}\right) & \text { if } \frac{1}{2}<q<1, \\ \sup _{t^{\prime}<t} N\left(x,\left(\frac{2^{p}-4}{3}\right)^{q} t^{\prime q}\right) & \text { if } 0<q<\frac{1}{2}\end{cases}
$$

where $p=1 / q$.

Proof. It follows from (2.1) and (N4) that

$$
N^{\prime}(f(0), t) \geq \min \left\{N\left(0,\left(\frac{t}{3}\right)^{q}\right), N\left(0,\left(\frac{t}{3}\right)^{q}\right), N\left(0,\left(\frac{t}{3}\right)^{q}\right)\right\}=1
$$

for all $t \in(0, \infty)$. By (N2), we have $f(0)=0$. We will prove the theorem in three cases, $\mathrm{q}>1, \frac{1}{2}<q<1$, and $0<q<\frac{1}{2}$.

Case 1. Let $q>1$ and let $J_{n} f: X \rightarrow Y$ be a mapping defined by

$$
J_{n} f(x)=\frac{1}{2}\left(4^{-n}\left(f\left(2^{n} x\right)+f\left(-2^{n} x\right)\right)+2^{-n}\left(f\left(2^{n} x\right)-f\left(-2^{n} x\right)\right)\right)
$$

for all $x \in X$. Notice that $J_{0} f(x)=f(x)$ and

$$
\begin{aligned}
J_{j} f(x)-J_{j+1} f(x)= & \frac{D f\left(2^{j} x, 2^{j} x,-2^{j} x\right)}{2 \cdot 4^{j+1}}+\frac{D f\left(-2^{j} x,-2^{j} x, 2^{j} x\right)}{2 \cdot 4^{j+1}} \\
& +\frac{D f\left(2^{j} x, 2^{j} x,-2^{j} x\right)}{2^{j+2}}-\frac{D f\left(-2^{j} x,-2^{j} x, 2^{j} x\right)}{2^{j+2}}
\end{aligned}
$$

for all $x \in X$ and $j \geq 0$. Together with (N3), (N4) and (2.1), this equation implies that if $n+m>m \geq 0$, then

$$
\begin{aligned}
& N^{\prime}\left(J_{m} f(x)-J_{n+m} f(x), \sum_{j=m}^{n+m-1} \frac{3}{2}\left(\frac{2^{p}}{2}\right)^{j} t^{p}\right) \\
& \quad=N^{\prime}\left(\sum_{j=m}^{n+m-1}\left(J_{j} f(x)-J_{j+1} f(x)\right), \sum_{j=m}^{n+m-1} \frac{3 \cdot 2^{j p}}{2^{j+1}} t^{p}\right) \\
& \quad \geq_{j=m_{, \ldots, n+m-1}}\left\{N^{\prime}\left(J_{j} f(x)-J_{j+1} f(x), \frac{3 \cdot 2^{j p}}{2^{j+1}} t^{p}\right)\right\} \\
& \quad \geq \min _{j=m_{, \ldots, n+m-1}}\left\{\operatorname { m i n } \left\{N^{\prime}\left(\frac{\left(2^{j+1}+1\right) D f\left(2^{j} x, 2^{j} x,-2^{j} x\right)}{2 \cdot 4^{j+1}}, \frac{3\left(2^{j+1}+1\right) 2^{j p} t^{p}}{2 \cdot 4^{j+1}}\right),\right.\right. \\
& \left.\left.\quad N^{\prime}\left(\frac{1-\left(2^{j+1}\right) D f\left(-2^{j} x,-2^{j} x, 2^{j} x\right)}{2 \cdot 4^{j+1}}, \frac{3\left(2^{j+1}-1\right) 2^{j p} t^{p}}{2 \cdot 4^{j+1}}\right)\right\}\right\} \\
& \quad=N(x, t)
\end{aligned}
$$


for all $x \in X$ and $t>0$. Let $\varepsilon>0$ be given. Since $\lim _{t \rightarrow \infty} N(x, t)=1$, there is $t_{0}>0$ such that

$$
N\left(x, t_{0}\right) \geq 1-\varepsilon
$$

We observe that for some $\tilde{t}>t_{0}$, the series $\sum_{j=0}^{\infty} \frac{3 \cdot 2^{j p}{ }^{j+1}}{2^{p}}$ converges for $p=\frac{1}{q}<1$. It guarantees that, for an arbitrary given $c>0$, there exists some $n_{0} \geq 0$ such that

$$
\sum_{j=m}^{n+m-1} \frac{3 \cdot 2^{j p}}{2^{j+1}} \tilde{t}^{p}<c
$$

for each $m \geq n_{0}$ and $n>0$. By (N5) and (2.4), we have

$$
\begin{aligned}
N^{\prime}\left(J_{m} f(x)-J_{n+m} f(x), c\right) & \geq N^{\prime}\left(J_{m} f(x)-J_{n+m} f(x), \sum_{j=m}^{n+m-1} \frac{3 \cdot 2^{j p}}{2^{j+1}} \tilde{t}^{p}\right) \\
& \geq N(x, \tilde{t}) \\
& \geq N\left(x, t_{0}\right) \\
& \geq 1-\varepsilon
\end{aligned}
$$

for all $x \in X$. Hence $\left\{J_{n} f(x)\right\}$ is a Cauchy sequence in the fuzzy Banach space $\left(Y, N^{\prime}\right)$, and so, we can define a mapping $F: X \rightarrow Y$ by

$$
F(x):=N^{\prime}-\lim _{n \rightarrow \infty} J_{n} f(x)
$$

for all $x \in X$. Moreover, if we put $m=0$ in (2.4), we have

$$
N^{\prime}\left(f(x)-J_{n} f(x), t\right) \geq N\left(x, \frac{t^{q}}{\left(\sum_{j=0}^{n-1} \frac{3 \cdot 2^{j p}}{2^{j+1}}\right)^{q}}\right)
$$

for all $x \in X$. Next we will show that $F$ is quadratic additive. Using (N4), we have

$$
\begin{aligned}
N^{\prime}(D F(x, y, z), t) \geq \min \{ & N^{\prime}\left(\left(F-J_{n} f\right)(x+y+z), \frac{t}{28}\right), N^{\prime}\left(\left(F-J_{n} f\right)(x), \frac{t}{28}\right), \\
& N^{\prime}\left(\left(F-J_{n} f\right)(y), \frac{t}{28}\right), N^{\prime}\left(\left(F-J_{n} f\right)(z), \frac{t}{28}\right) \\
& N^{\prime}\left(\left(J_{n} f-F\right)(x+y), \frac{t}{28}\right), N^{\prime}\left(\left(J_{n} f-F\right)(x+z), \frac{t}{28}\right), \\
& \left.N^{\prime}\left(\left(J_{n} f-F\right)(y+z), \frac{t}{28}\right), N^{\prime}\left(D J_{n} f(x, y, z), \frac{3 t}{4}\right)\right\}
\end{aligned}
$$

for all $x, y, z \in X$ and $n \in \mathbb{N}$. The first seven terms on the right-hand side of (2.6) tend to 1 as $n \rightarrow \infty$ by the definition of $F$ and (N2), and the last term holds

$$
\begin{aligned}
& N^{\prime}\left(D J_{n} f(x, y, z), \frac{3 t}{4}\right) \\
& \geq \min \left\{N^{\prime}\left(\frac{D f\left(2^{n} x, 2^{n} y, 2^{n} z\right)}{2 \cdot 4^{n}}, \frac{3 t}{16}\right), N^{\prime}\left(\frac{D f\left(-2^{n} x,-2^{n} y,-2^{n} z\right)}{2 \cdot 4^{n}}, \frac{3 t}{16}\right),\right. \\
& \left.N^{\prime}\left(\frac{D f\left(2^{n} x, 2^{n} y, 2^{n} z\right)}{2 \cdot 2^{n}}, \frac{3 t}{16}\right), N^{\prime}\left(\frac{D f\left(-2^{n} x,-2^{n} y,-2^{n} z\right)}{2 \cdot 2^{n}}, \frac{3 t}{16}\right)\right\}
\end{aligned}
$$


for all $x, y, z \in X$. By (N3) and (2.1), we obtain

$$
\begin{aligned}
& N^{\prime}\left(\frac{D f\left( \pm 2^{n} x, \pm 2^{n} y, \pm 2^{n} z\right)}{2 \cdot 4^{n}}, \frac{3 t}{16}\right) \\
& \quad=N^{\prime}\left(D f\left( \pm 2^{n} x, \pm 2^{n} y, \pm 2^{n} z\right), \frac{3 \cdot 4^{n} t}{8}\right) \\
& \quad \geq \min \left\{N\left(2^{n} x,\left(\frac{4^{n} t}{8}\right)^{q}\right), N\left(2^{n} y,\left(\frac{4^{n} t}{8}\right)^{q}\right), N\left(2^{n} z,\left(\frac{4^{n} t}{8}\right)^{q}\right)\right\} \\
& \quad \geq \min \left\{N\left(x, 2^{(2 q-1) n-3 q} t^{q}\right), N\left(y, 2^{(2 q-1) n-3 q} t^{q}\right), N\left(z, 2^{(2 q-1) n-3 q} t^{q}\right)\right\}
\end{aligned}
$$

and

$$
\begin{aligned}
& N^{\prime}\left(\frac{D f\left( \pm 2^{n} x, \pm 2^{n} y, \pm 2^{n} z\right)}{2 \cdot 2^{n}}, \frac{3 t}{16}\right) \\
& \quad \geq \min \left\{N\left(x, 2^{(q-1) n-3 q} t^{q}\right), N\left(y, 2^{(q-1) n-3 q} t^{q}\right), N\left(z, 2^{(q-1) n-3 q} t^{q}\right)\right\}
\end{aligned}
$$

for all $x, y, z \in X$ and $n \in \mathbb{N}$. Since $q>1$, together with (N5), we can deduce that the last term of (2.6) also tends to 1 as $n \rightarrow \infty$. It follows from (2.6) that

$$
N^{\prime}(D F(x, y, z), t)=1
$$

for all $x, y, z \in X$ and $t>0$. By (N2), this means that $D F(x, y, z)=0$ for all $x, y, z \in$ $X$.

Now we approximate the difference between $f$ and $F$ in a fuzzy sense. For an arbitrary fixed $x \in X$ and $t>0$, choose $0<\varepsilon<1$ and $0<t^{\prime}<t$. Since $F$ is the limit of $\left\{J_{n} f\right.$ $(x)\}$, there is $n \in \mathbb{N}$ such that

$$
N^{\prime}\left(F(x)-J_{n} f(x), t-t^{\prime}\right) \geq 1-\varepsilon .
$$

By (2.5), we have

$$
\begin{aligned}
N^{\prime}(F(x)-f(x), t) & \geq \min \left\{N^{\prime}\left(F(x)-J_{n} f(x), t-t^{\prime}\right), N^{\prime}\left(J_{n} f(x)-f(x), t^{\prime}\right)\right\} \\
& \geq \min \left\{1-\varepsilon, N\left(x, \frac{t^{\prime q}}{\left(\sum_{j=0}^{n-1} \frac{3 \cdot 2^{j p}}{2^{j+1}}\right)^{q}}\right)\right\} \\
& \geq \min \left\{1-\varepsilon, N\left(x,\left(\frac{\left(2-2^{p}\right) t^{\prime}}{3}\right)^{q}\right)\right\} .
\end{aligned}
$$

Because $0<\varepsilon<1$ is arbitrary, we get the inequality (2.2) in this case.

Finally, to prove the uniqueness of $F$, let $F^{\prime}: X \rightarrow Y$ be another quadratic-additive mapping satisfying (2.2). Then by (2.3), we get

$$
\left\{\begin{array}{l}
F(x)-J_{n} F(x)=\sum_{j=0}^{n-1}\left(J_{j} F(x)-J_{j+1} F(x)\right)=0 \\
F^{\prime}(x)-J_{n} F^{\prime}(x)=\sum_{j=0}^{n-1}\left(J_{j} F^{\prime}(x)-J_{j+1} F^{\prime}(x)\right)=0
\end{array}\right.
$$


for all $x \in X$ and $n \in \mathbb{N}$. Together with (N4) and (2.2), this implies that

$$
\begin{aligned}
& N^{\prime}(F(x)-\left.F^{\prime}(x), t\right) \\
&= N^{\prime}\left(J_{n} F(x)-J_{n} F^{\prime}(x), t\right) \\
& \geq \min \left\{N^{\prime}\left(J_{n} F(x)-J_{n} f(x), \frac{t}{2}\right), N^{\prime}\left(J_{n} f(x)-J_{n} F^{\prime}(x), \frac{t}{2}\right)\right\} \\
& \geq \min \left\{N^{\prime}\left(\frac{(F-f)\left(2^{n} x\right)}{2 \cdot 4^{n}}, \frac{t}{8}\right), N^{\prime}\left(\frac{\left(f-F^{\prime}\right)\left(2^{n} x\right)}{2 \cdot 4^{n}}, \frac{t}{8}\right),\right. \\
& N^{\prime}\left(\frac{(F-f)\left(-2^{n} x\right)}{2 \cdot 4^{n}}, \frac{t}{8}\right), N^{\prime}\left(\frac{\left(f-F^{\prime}\right)\left(-2^{n} x\right)}{2 \cdot 4^{n}}, \frac{t}{8}\right), \\
& N^{\prime}\left(\frac{(F-f)\left(2^{n} x\right)}{2 \cdot 2^{n}}, \frac{t}{8}\right), N^{\prime}\left(\frac{\left(f-F^{\prime}\right)\left(2^{n} x\right)}{2 \cdot 2^{n}}, \frac{t}{8}\right), \\
&\left.N^{\prime}\left(\frac{(F-f)\left(-2^{n} x\right)}{2 \cdot 2^{n}}, \frac{t}{8}\right), N^{\prime}\left(\frac{\left(f-F^{\prime}\right)\left(-2^{n} x\right)}{2 \cdot 2^{n}}, \frac{t}{8}\right)\right\} \\
& \geq \sup _{t^{\prime}<t} N\left(x, 2^{(q-1) n-2 q}\left(\frac{2-2^{p}}{3}\right)^{q} t^{\prime q}\right)
\end{aligned}
$$

for all $x \in X$ and $n \in \mathbb{N}$. Observe that, for $q=\frac{1}{p}>1$, the last term of the above inequality tends to 1 as $n \rightarrow \infty$ by (N5). This implies that $N^{\prime}\left(F(x)-F^{\prime}(x), t\right)=1$, and so, we get

$$
F(x)=F^{\prime}(x)
$$

for all $x \in X$ by (N2).

Case 2. Let $\frac{1}{2}<q<1$ and let $J_{n} f: X \rightarrow Y$ be a mapping defined by

$$
J_{n} f(x)=\frac{1}{2}\left(4^{-n}\left(f\left(2^{n} x\right)+f\left(-2^{n} x\right)\right)+2^{n}\left(f\left(\frac{x}{2^{n}}\right)-f\left(-\frac{x}{2^{n}}\right)\right)\right)
$$

for all $x \in X$. Then we have $J_{0} f(x)=f(x)$ and

$$
\begin{aligned}
J_{j} f(x)-J_{j+1} f(x)= & \frac{D f\left(-2^{j} x,-2^{j} x, 2^{j} x\right)}{2 \cdot 4^{j+1}}+\frac{D f\left(2^{j} x, 2^{j} x,-2^{j} x\right)}{2 \cdot 4^{j+1}} \\
& -2^{j-1}\left(D f\left(\frac{x}{2^{j+1}}, \frac{x}{2^{j+1}}, \frac{-x}{2^{j+1}}\right)-D f\left(\frac{-x}{2^{j+1}}, \frac{-x}{2^{j+1}}, \frac{x}{2^{j+1}}\right)\right)
\end{aligned}
$$

for all $x \in X$ and $j \geq 0$. If $n+m>m \geq 0$, then we have

$$
\begin{gathered}
N^{\prime}\left(J_{m} f(x)-J_{n+m} f(x), \sum_{j=m}^{n+m-1}\left(\frac{3}{4}\left(\frac{2^{p}}{4}\right)^{j}+\frac{3}{2^{p}}\left(\frac{2}{2^{p}}\right)^{j}\right) t^{p}\right) \\
\geq \min _{j=m, \ldots, n+m-1}\left\{\operatorname { m i n } \left\{N^{\prime}\left(\frac{D f\left(2^{j} x, 2^{j} x,-2^{j} x\right)}{2 \cdot 4^{j+1}}, \frac{3 \cdot 2^{j p} t^{p}}{2 \cdot 4^{j+1}}\right),\right.\right. \\
N^{\prime}\left(\frac{D f\left(-2^{j} x,-2^{j} x, 2^{j} x\right)}{2 \cdot 4^{j+1}}, \frac{3 \cdot 2^{j p} t^{p}}{2 \cdot 4^{j+1}}\right), \\
N^{\prime}\left(-2^{j-1} D f\left(\frac{x}{2^{j+1}}, \frac{x}{2^{j+1}}, \frac{-x}{2^{j+1}}\right), \frac{3 \cdot 2^{j-1} t^{p}}{2^{(j+1)^{p}}}\right), \\
\left.\left.N^{\prime}\left(2^{j^{j-1}} D f\left(\frac{-x}{2^{j+1}}, \frac{-x}{2^{j+1}}, \frac{x}{2^{j+1}}\right), \frac{3 \cdot 2^{j-1} t^{p}}{2^{(j+1)^{p}}}\right)\right\}\right\} \\
\geq \min _{j=m, \ldots, n+m-1}\left\{N\left(2^{j} x, 2^{j} t\right), N\left(\frac{x}{2^{j+1}}, \frac{t}{2^{j+1}}\right)\right\} \\
=N(x, t)
\end{gathered}
$$


for all $x \in X$ and $t>0$. In the similar argument following (2.4) of the previous case, we can define the limit $F(x):=N^{\prime}-\lim _{n \rightarrow \infty} J_{n} f(x)$ of the Cauchy sequence $\left\{_{n} f(x)\right\}$ in the Banach fuzzy space $Y$. Moreover, putting $m=0$ in the above inequality, we have

$$
N^{\prime}\left(f(x)-J_{n} f(x), t\right) \geq N\left(x, \frac{t^{q}}{\left(\sum_{j=0}^{n-1}\left(\frac{3}{4}\left(\frac{2^{p}}{4}\right)^{j}+\frac{3}{2^{p}}\left(\frac{2}{2^{p}}\right)^{j}\right)\right)^{q}}\right)
$$

for each $x \in X$ and $t>0$. To prove that $F$ is a quadratic-additive function, we have enough to show that the last term of (2.6) in Case 1 tends to 1 as $n \rightarrow \infty$. By (N3) and (2.1), we get

$$
\begin{aligned}
& N^{\prime}\left(D J_{n} f(x, y, z), \frac{3 t}{4}\right) \\
& \geq \min \left\{N^{\prime}\left(\frac{D f\left(2^{n} x, 2^{n} y, 2^{n} z\right)}{2 \cdot 4^{n}}, \frac{3 t}{16}\right), N^{\prime}\left(\frac{D f\left(-2^{n} x,-2^{n} y,-2^{n} z\right)}{2 \cdot 4^{n}}, \frac{3 t}{16}\right),\right. \\
& \left.N^{\prime}\left(2^{n-1} D f\left(\frac{x}{2^{n}}, \frac{y}{2^{n}}, \frac{z}{2^{n}}\right), \frac{3 t}{16}\right), N^{\prime}\left(2^{n-1} D f\left(\frac{-x}{2^{n}}, \frac{-y}{2^{n}}, \frac{-z}{2^{n}}\right), \frac{3 t}{16}\right)\right\} \\
& \geq \min \left\{N\left(x, 2^{(2 q-1) n-3 q} t^{q}\right), N\left(y, 2^{(2 q-1) n-3 q} t^{q}\right), N\left(z, 2^{(2 q-1) n-3 q} t^{q}\right),\right. \\
& \left.N\left(x, 2^{(1-q) n-3 q} t^{q}\right), N\left(y, 2^{(1-q) n-3 q} t^{q}\right), N\left(z, 2^{(1-q) n-3 q} t^{q}\right)\right\}
\end{aligned}
$$

for each $x, y, z \in X$ and $t>0$. Observe that all the terms on the right-hand side of the above inequality tend to 1 as $n \rightarrow \infty$, since $\frac{1}{2}<q<1$. Hence, together with the similar argument after (2.6), we can say that $D F(x, y, z)=0$ for all $x, y, z \in X$. Recall, in Case 1, the inequality (2.2) follows from (2.5). By the same reasoning, we get (2.2) from (2.8) in this case. Now to prove the uniqueness of $F$, let $F^{\prime}$ be another quadraticadditive mapping satisfying (2.2). Then, together with (N4), (2.2), and (2.7), we have

$$
\begin{aligned}
& N^{\prime}\left(F(x)-F^{\prime}(x), t\right) \\
& =N^{\prime}\left(J_{n} F(x)-J_{n} F^{\prime}(x), t\right) \\
& \geq \min \left\{N^{\prime}\left(J_{n} F(x)-J_{n} f(x), \frac{t}{2}\right), N^{\prime}\left(J_{n} f(x)-J_{n} F^{\prime}(x), \frac{t}{2}\right)\right\} \\
& \geq \min \left\{N^{\prime}\left(\frac{(F-f)\left(2^{n} x\right)}{2 \cdot 4^{n}}, \frac{t}{8}\right),\left(\frac{\left(f-F^{\prime}\right)\left(2^{n} x\right)}{2 \cdot 4^{n}}, \frac{t}{8}\right),\right. \\
& N^{\prime}\left(\frac{(F-f)\left(-2^{n} x\right)}{2 \cdot 4^{n}}, \frac{t}{8}\right), N^{\prime}\left(\frac{\left(f-F^{\prime}\right)\left(-2^{n} x\right)}{2 \cdot 4^{n}}, \frac{t}{8}\right), \\
& N^{\prime}\left(2^{n-1}\left((F-f)\left(\frac{x}{2^{n}}\right)\right), \frac{t}{8}\right), N^{\prime}\left(2^{n-1}\left(\left(f-F^{\prime}\right)\left(\frac{x}{2^{n}}\right)\right), \frac{t}{8}\right), \\
& \left.N^{\prime}\left(2^{n-1}\left((F-f)\left(\frac{-x}{2^{n}}\right)\right), \frac{t}{8}\right), N^{\prime}\left(2^{n-1}\left(\left(f-F^{\prime}\right)\left(\frac{-x}{2^{n}}\right)\right), \frac{t}{8}\right)\right\} \\
& \geq \min \left\{\sup _{t^{\prime}<t} N\left(x, 2^{(2 q-1) n-2 q}\left(\frac{\left(4-2^{p}\right)\left(2^{p}-2\right)}{6}\right)^{q} t^{q q}\right)\right. \text {, } \\
& \left.\sup _{t^{\prime}<t} N\left(x, 2^{(1-q) n-2 q}\left(\frac{\left(4-2^{p}\right)\left(2^{p}-2\right)}{6}\right)^{q} t^{\prime q}\right)\right\}
\end{aligned}
$$

for all $x \in X$ and $n \in \mathbb{N}$. Since $\lim _{n \rightarrow \infty} 2^{(2 q-1) n-2 q}=\lim _{n \rightarrow \infty} 2^{(1-q) n-2 q}=\infty$ in this case, both terms on the right-hand side of the above inequality tend to 1 as $n \rightarrow \infty$ by (N5). This implies that $N^{\prime}\left(F(x)-F^{\prime}(x), t\right)=1$ and so $F(x)=F^{\prime}(x)$ for all $x \in X$ by (N2). 
Case 3. Finally, we take $0<q<\frac{1}{2}$ and define $J_{n} f: X \rightarrow Y$ by

$$
J_{n} f(x)=\frac{1}{2}\left(4^{n}\left(f\left(2^{-n} x\right)+f\left(-2^{-n} x\right)\right)+2^{n}\left(f\left(\frac{x}{2^{n}}\right)-f\left(-\frac{x}{2^{n}}\right)\right)\right)
$$

for all $x \in X$. Then we have $J_{0} f(x)=f(x)$ and

$$
\begin{aligned}
J_{j} f(x)-J_{j+1} f(x)= & -\frac{4^{j}}{2}\left(D f\left(\frac{-x}{2^{j+1}}, \frac{-x}{2^{j+1}}, \frac{x}{2^{j+1}}\right)+D f\left(\frac{x}{2^{j+1}}, \frac{x}{2^{j+1}}, \frac{-x}{2^{j+1}}\right)\right) \\
& -2^{j-1}\left(D f\left(\frac{x}{2^{j+1}}, \frac{x}{2^{j+1}}, \frac{-x}{2^{j+1}}\right)-D f\left(\frac{-x}{2^{j+1}}, \frac{-x}{2^{j+1}}, \frac{x}{2^{j+1}}\right)\right)
\end{aligned}
$$

which implies that if $n+m>m \geq 0$, then

$$
\begin{aligned}
& N^{\prime}\left(J_{m} f(x)-J_{n+m} f(x), \sum_{j=m}^{n+m-1} \frac{3}{2^{p}}\left(\frac{4}{2^{p}}\right)^{j} t^{p}\right) \\
& \geq \min _{j=m, \ldots, n+m-1}\left\{\operatorname { m i n } \left\{N^{\prime}\left(-\frac{\left(4^{j}+2^{j}\right) D f\left(\frac{x}{2^{j+1}}, \frac{x}{2^{j+1}}, \frac{-x}{2^{j+1}}\right)}{2}, \frac{3\left(4^{j}+2^{j}\right) t^{p}}{2 \cdot 2^{(j+1)^{p}}}\right),\right.\right. \\
& \left.\left.\quad N^{\prime}\left(-\frac{\left(4^{j}-2^{j}\right) D f\left(\frac{-x}{2^{j+1}}, \frac{-x}{2^{j+1}}, \frac{x}{2^{j+1}}\right)}{2}, \frac{3\left(4^{j}-2^{j}\right) t^{p}}{2 \cdot 2^{(j+1)^{p}}}\right)\right\}\right\} \\
& \geq \min _{j=m, \ldots, n+m-1}\left\{N\left(\frac{x}{2^{j+1}}, \frac{t}{2^{j+1}}\right)\right\}=N(x, t)
\end{aligned}
$$

for all $x \in X$ and $t>0$. Similar to the previous cases, it leads us to define the mapping $F: X \rightarrow Y$ by $F(x):=N^{\prime}-\lim _{n \rightarrow \infty} J_{n} f(x)$. Putting $m=0$ in the above inequality, we have

$$
N^{\prime}\left(f(x)-J_{n} f(x), t\right) \geq N\left(x, \frac{t^{q}}{\left(\sum_{j=0}^{n-1} \frac{3}{2^{p}}\left(\frac{4}{2^{p}}\right)^{j}\right)^{q}}\right)
$$

for all $x \in X$ and $t>0$. Notice that

$$
\begin{aligned}
& N^{\prime}\left(D J_{n} f(x, y, z), \frac{3 t}{4}\right) \\
& \geq \min \left\{N^{\prime}\left(\frac{4^{n}}{2} D f\left(\frac{x}{2^{n}}, \frac{y}{2^{n^{\prime}}}, \frac{z}{2^{n}}\right), \frac{3 t}{16}\right), N^{\prime}\left(\frac{4^{n}}{2} D f\left(\frac{-x}{2^{n}}, \frac{-y}{2^{n}}, \frac{-z}{2^{n}}\right), \frac{3 t}{16}\right),\right. \\
& \left.N^{\prime}\left(2^{n-1} D f\left(\frac{x}{2^{n}}, \frac{y}{2^{n}}, \frac{z}{2^{n}}\right), \frac{3 t}{16}\right), N^{\prime}\left(2^{n-1} D f\left(\frac{-x}{2^{n}}, \frac{-y}{2^{n}}, \frac{-z}{2^{n}}\right), \frac{3 t}{16}\right)\right\} \\
& \geq \min \left\{N\left(x, 2^{(1-2 q) n-3 q} t^{q}\right), N\left(y, 2^{(1-2 q) n-3 q} t^{q}\right), N\left(z, 2^{(1-2 q) n-3 q} t^{q}\right),\right. \\
& \left.N\left(x, 2^{(1-q) n-3 q} t^{q}\right), N\left(y, 2^{(1-q) n-3 q} t^{q}\right), N\left(z, 2^{(1-q) n-3 q} t^{q}\right)\right\}
\end{aligned}
$$

for each $x, y, z \in X$ and $t>0$. Since $0<q<\frac{1}{2}$, all terms on the right-hand side tend to 1 as $n \rightarrow \infty$, which implies that the last term of (2.6) tends to 1 as $n \rightarrow \infty$. Therefore, we can say that $D F \equiv 0$. Moreover, using the similar argument after (2.6) in Case 1 , we get the inequality (2.2) from (2.9) in this case. To prove the uniqueness of $F$, let $F^{\prime}: X \rightarrow Y$ be another quadratic-additive function satisfying (2.2). Then by (2.7), we get 


$$
\begin{aligned}
& N^{\prime}(F(x)-\left.F^{\prime}(x), t\right) \\
& \geq \min \left\{N^{\prime}\left(J_{n} F(x)-J_{n} f(x), \frac{t}{2}\right), N^{\prime}\left(J_{n} f(x)-J_{n} F^{\prime}(x), \frac{t}{2}\right)\right\} \\
& \geq \min \left\{N^{\prime}\left(\frac{4^{n}}{2}\left((F-f)\left(\frac{x}{2^{n}}\right)\right), \frac{t}{8}\right), \frac{4^{n}}{2}\left(\left(f-F^{\prime}\right)\left(\frac{x}{2^{n}}\right)\right), \frac{t}{8}\right), \\
& N^{\prime}\left(\frac{4^{n}}{2}\left((F-f)\left(-\frac{x}{2^{n}}\right)\right), \frac{t}{8}\right), N^{\prime}\left(\frac{4^{n}}{2}\left(\left(f-F^{\prime}\right)\left(-\frac{x}{2^{n}}\right)\right), \frac{t}{8}\right), \\
& N^{\prime}\left(2^{n-1}\left((F-f)\left(\frac{x}{2^{n}}\right)\right), \frac{t}{8}\right), N^{\prime}\left(2^{n-1}\left(\left(f-F^{\prime}\right)\left(\frac{x}{2^{n}}\right)\right), \frac{t}{8}\right), \\
&\left.N^{\prime}\left(2^{n-1}\left((F-f)\left(\frac{-x}{2^{n}}\right)\right), \frac{t}{8}\right), N^{\prime}\left(2^{n-1}\left(\left(f-F^{\prime}\right)\left(\frac{-x}{2^{n}}\right)\right), \frac{t}{8}\right)\right\} \\
& \geq \sup _{t^{\prime}<t} N\left(x, 2^{(1-2 q) n-2 q}\left(\frac{2^{p}-4}{3}\right) t^{q}\right)
\end{aligned}
$$

for all $x \in X$ and $n \in \mathbb{N}$. Observe that, for $0<q<\frac{1}{2}$, the last term tends to 1 as $n \rightarrow$ $\infty$ by (N5). This implies that $N^{\prime}\left(F(x)-F^{\prime}(x), t\right)=1$ and $F(x)=F^{\prime}(x)$ for all $x \in X$ by (N2).

Remark 2.3. Consider a mapping $f: X \rightarrow Y$ satisfying (2.1) for all $x, y, z \in X$ and a real number $q<0$. Take any $t>0$. If we choose a real number $s$ with $0<3 s<t$, then we have

$$
N^{\prime}(D f(x, y, z), t) \geq N^{\prime}(D f(x, y, z), 3 s) \geq \min \left\{N\left(x, s^{q}\right), N\left(y, s^{q}\right), N\left(z, s^{q}\right)\right\}
$$

for all $x, y, z \in X$. Since $q<0$, we have $\lim _{s \rightarrow 0}+s^{q}=\infty$. This implies that

$$
\lim _{s \rightarrow 0^{+}} N\left(x, s^{q}\right)=\lim _{s \rightarrow 0^{+}} N\left(y, s^{q}\right)=\lim _{z \rightarrow 0^{+}} N\left(x, s^{q}\right)=1
$$

and so

$$
N^{\prime}(D f(x, y, z), t)=1
$$

for all $x, y, z \in X$ and $t>0$. By (N2), it allows us to get $D f(x, y, z)=0$ for all $x, y, z \in$ $X$. In other words, $f$ is itself a quadratic-additive mapping if $f$ is a fuzzy $q$-almost quadratic-additive mapping for the case $q<0$.

Corollary 2.4. Let $f$ be an even mapping satisfying all of the conditions of Theorem 2.2. Then there is a unique quadratic mapping $F: X \rightarrow Y$ such that

$$
N^{\prime}(F(x)-f(x), t) \geq \sup _{t^{\prime}<t} N\left(x,\left(\frac{\left|4-2^{p}\right| t^{\prime}}{3}\right)^{q}\right)
$$

for all $x \in X$ and $t>0$, where $p=1 / q$.

Proof. Let $J_{n} f$ be defined as in Theorem 2.2. Since $f$ is an even mapping, we obtain

$$
J_{n} f(x)= \begin{cases}\frac{f\left(2^{n} x\right)+f\left(-2^{n} x\right)}{2 \cdot 4^{n}} & \text { if } q>\frac{1}{2} \\ \frac{1}{2}\left(4^{n}\left(f\left(2^{-n} x\right)+f\left(-2^{-n} x\right)\right)\right) & \text { if } 0<q<\frac{1}{2}\end{cases}
$$

for all $x \in X$. Notice that $J_{0} f(x)=f(x)$ and

$$
J_{j} f(x)-J_{j+1} f(x)= \begin{cases}\frac{D f\left(2^{j} x, 2^{j} x,-2^{j} x\right)}{2 \cdot 4^{j+1}}+\frac{D f\left(-2^{j} x,-2^{j} x, 2^{j} x\right)}{2.4^{j+1}} & \text { if } q>\frac{1}{2}, \\ -\frac{4^{j}}{2}\left(D f\left(\frac{-x}{2^{j+1}}, \frac{-x}{2^{j+1}}, \frac{x}{2^{j+1}}\right)\right. & \text { if } 0<q<\frac{1}{2} \\ \left.+D f\left(\frac{x}{2^{j+1}}, \frac{j}{2^{j+1}}, \frac{-x}{2^{j+1}}\right)\right) & \text {. }\end{cases}
$$


for all $x \in X$ and $j \in \mathbb{N} \cup\{0\}$. From these, using the similar method in Theorem 2.2, we obtain the quadratic-additive function $F$ satisfying (2.10). Notice that $F(x):=N^{\prime}$ $\lim _{n \rightarrow \infty} J_{n} f(x)$ for all $x \in X, F$ is even, and $D F(x, y, z)=0$ for all $x, y, z \in X$. Hence, we get

$$
F(x+y)+F(x-y)-2 F(x)-2 F(y)=-D F(x, y,-x)=0
$$

for all $x, y \in X$. This means that $F$ is a quadratic mapping.

Corollary 2.5. Let $f$ be an odd mapping satisfying all of the conditions of Theorem 2.2. Then there is a unique additive mapping $F: X \rightarrow Y$ such that

$$
N^{\prime}(F(x)-f(x), t) \geq \sup _{t^{\prime}<t} N\left(x,\left(\frac{\left|2-2^{p}\right| t^{\prime}}{3}\right)^{q}\right)
$$

for all $x \in X$ and $t>0$, where $p=1 / q$.

Proof. Let $J_{n} f$ be defined as in Theorem 2.2. Since $f$ is an odd mapping, we obtain

$$
J_{n} f(x)= \begin{cases}\frac{f\left(2^{n} x\right)+f\left(-2^{n} x\right)}{2^{n+1}} & \text { if } q>1, \\ 2^{n-1}\left(f\left(2^{-n} x\right)+f\left(-2^{-n} x\right)\right) & \text { if } 0<q<1\end{cases}
$$

for all $x \in X$. Notice that $J_{0} f(x)=f(x)$ and

$$
J_{j} f(x)-J_{j+1} f(x)=\left\{\begin{array}{cl}
\frac{D f\left(2^{j} x, 2^{j} x,-2^{j} x\right)}{-2^{2^{j+1}}\left(D f\left(\frac{x}{2^{j+1}}, \frac{D f\left(-2^{j} x,-2^{j} x, 2^{j} x\right)}{2^{j+1}}, \frac{-x}{2^{j+2}}\right)\right.} & \text { if } q i 1, \\
\left.-D f\left(\frac{-x}{2^{j+1}}, \frac{-x}{2^{j+1}}, \frac{x}{2^{j+1}}\right)\right) & \text { if } 0 ; q<1
\end{array}\right.
$$

for all $x \in X$ and $j \in \mathbb{N} \cup\{0\}$. From these, using the similar method in Theorem 2.2, we obtain the quadratic-additive function $F$ satisfying (2.11). Notice that $F(x):=N^{\prime}$ $\lim _{n \rightarrow \infty} J_{n} f(x)$ for all $x \in X, F$ is odd, $F(2 x)=2 F(x)$, and $D F(x, y, z)=0$ for all $x, y$, $z \in X$. Hence, we get

$$
F(x+y)-F(x)-F(y)=D F\left(\frac{x-y}{2}, \frac{x+y}{2}, \frac{-x+y}{2}\right)=0
$$

for all $x, y \in X$. This means that $F$ is an additive mapping.

We can use Theorem 2.2 to get a classical result in the framework of normed spaces. Let $(X,\|\cdot\|)$ be a normed linear space. Then we can define a fuzzy norm $N_{X}$ on $X$ by following

$$
N_{X}(x, t)= \begin{cases}0, & t \leq\|x\| \\ 1, & t>\|x\|\end{cases}
$$

where $x \in X$ and $t \in \mathbb{R}$, see [14]. Suppose that $f: X \rightarrow Y$ is a mapping into a Banach space $(Y, \||\cdot| \mid)$ such that

$$
\|\mid D f(x, y, z)\|\|\leq\| x\left\|^{p}+\right\| y\left\|^{p}+\right\| z \|^{p}
$$

for all $x, y, z \in X$, where $p>0$ and $p \neq 1,2$. Let $N_{Y}$ be a fuzzy norm on $Y$. Then we get

$$
N_{Y}(D f(x, y, z), s+t+u)= \begin{cases}0, & s+t+u \leq\|\| D f(x, y, z)\|\| \\ 1, & s+t+u>\|D f(x, y, z)\| \|\end{cases}
$$

for all $x, y, z \in X$ and $s, t, u \in \mathbb{R}$. Consider the case $N_{Y}(D f(x, y, z), s+t+u)=0$. This implies that 


$$
\|x\|^{p}+\|y\|^{p}+\|z\|^{p} \geq|\|D f(x, y, z) \mid\| \geq s+t+u
$$

and so either $\|x\|^{p} \geq s$ or $\|y\|^{p} \geq t$ or $\|z\|^{p} \geq u$ in this case. Hence, for $q=\frac{1}{p}$, we have

$$
\min \left\{N_{X}\left(x, s^{q}\right), N_{X}\left(y, t^{q}\right), N_{X}\left(z, u^{q}\right)\right\}=0
$$

for all $x, y, z \in X$ and $s, t, u>0$. Therefore, in every case, the inequality

$$
N_{Y}(D f(x, y, z), s+t+u) \geq \min \left\{N_{X}\left(x, s^{q}\right), N_{X}\left(y, t^{q}\right), N_{X}\left(z, u^{q}\right)\right\}
$$

holds. It means that $f$ is a fuzzy $q$-almost quadratic-additive mapping, and by Theorem 2.2, we get the following stability result.

Corollary 2.6. Let $(X,\|\cdot\|)$ be a normed linear space and let $(Y,\||\cdot|\|)$ be a Banach space. If $f: X \rightarrow Y$ satisfies

$$
\|\mid D f(x, y, z)\| \leq \leq\|x\|^{p}\|y\|^{p}+\|z\|^{p}
$$

for all $x, y, z \in X$, where $p>0$ and $p \neq 1,2$, then there is a unique quadratic-additive mapping $F: X \rightarrow Y$ such that

$$
\|F(x)-f(x)\| \leq \leq \begin{cases}\frac{3}{2-2^{p}} \|\left. x\right|^{p} & \text { if } p<1, \\ \frac{6}{\left(2-2^{p}\right)\left(4-2^{p}\right)}|| x||^{p} & \text { if } 1<p<2, \\ \left.\frac{3}{2^{p}-4}|| x\right|^{p} & \text { if } 2<p\end{cases}
$$

for all $x \in X$.

\section{Authors' contributions}

All authors carried out the proof. All authors conceived of the study, and participated in its design and coordination. All authors read and approved the final manuscript.

\section{Competing interests}

The authors declare that they have no competing interests.

Received: 15 March 2011 Accepted: 25 September 2011 Published: 25 September 2011

\section{References}

1. Ulam, SM: A collection of mathematical problems. Interscience, New York. (1968)

2. Hyers, DH: On the stability of the linear functional equation. Proc Natl Acad Sci USA. 27, 222-224 (1941). doi:10.1073/ pnas.27.4.222

3. Aoki, T: On the stability of the linear transformation in Banach spaces. J Math Soc Japan. 2, 64-66 (1950). doi:10.2969/ jmsj/00210064

4. Rassias, ThM: On the stability of the linear mapping in Banach spaces. Proc Amer Math Soc. 72, $297-300$ (1978). doi:10.1090/S0002-9939-1978-0507327-1

5. Czerwik, S: Functional equations and inequalities in several variables. World Scientific, Singapore. (2002)

6. Czerwik, S: On the stability of the quadratic mapping in normed spaces. Abh Math Sem Univ Hamburg. 62, 59-64 (1992). doi:10.1007/BF02941618

7. Găvruta, P: A generalization of the Hyers-Ulam-Rassias stability of approximately additive mappings. J Math Anal Appl. 184, 431-436 (1994). doi:10.1006/jmaa.1994.1211

8. Hyers, DH, Isac, G, Rassias, ThM: Stability of functional equations in several variables. Birkhäuser, Boston. (1998)

9. Jung, S-M: Hyers-Ulam-Rassias stability of functional equations in nonlinear analysis. Springer, New York. (2011)

10. Kim, G-H: On the stability of functional equations with square-symmetric operation. Math Inequal Appl. 4, 257-266 (2001)

11. Kim, H-M: On the stability problem for a mixed type of quartic and quadratic functional equation. J Math Anal Appl. 324, 358-372 (2006). doi:10.1016/j.jmaa.2005.11.053

12. Lee, Y-H: On the Hyers-Ulam-Rassias stability of the generalized polynomial function of degree 2. J Chuncheong Math Soc. 22, 201-209 (2009)

13. Lee, Y-H: On the stability of the monomial functional equation. Bull Korean Math Soc. 45, 397-403 (2008). doi:10.4134/ BKMS.2008.45.2.397

14. Lee, Y-H, Jun, K-W: A generalization of the Hyers-Ulam-Rassias stability of Jensen's equation. J Math Anal Appl. 238 , 305-315 (1999). doi:10.1006/jmaa.1999.6546

15. Lee, Y-H, Jun, K-W: A generalization of the Hyers-Ulam-Rassias stability of Pexider equation. J Math Anal Appl. 246, 627-638 (2000). doi:10.1006/jmaa.2000.6832 
16. Lee, YH, Jun, KW: On the stability of approximately additive mappings. Proc Amer Math Soc. 128, 1361-1369 (2000). doi:10.1090/50002-9939-99-05156-4

17. Skof, F: Local properties and approximations of operators. Rend Sem Mat Fis Milano. 53, 113-129 (1983). doi:10.1007/ BF02924890

18. Katsaras, AK: Fuzzy topological vector spaces II. Fuzzy Sets and System. 12, 143-154 (1984). doi:10.1016/0165-0114(84) 90034-4

19. Bag, T, Samanta, SK: Finite dimensional fuzzy normed linear spaces. J fuzzy Math. 11(3):687-705 (2003)

20. Cheng, SC, Mordeson, JN: Fuzzy linear operator and fuzzy normed linear spaces. Bull Calcutta Math Soc. 86, 429-436 (1994)

21. Kramosil, I, Michalek, J: Fuzzy metric and statistical metric spaces. Kyber-netica. 11, 326-334 (1975)

22. Mirmostafaee, AK, Moslehian, MS: Fuzzy versions of Hyers-Ulam-Rassias theorem. Fuzzy Sets and Systems. 159, 720-729 (2008). doi:10.1016/j.fss.2007.09.016

23. Mirmostafaee, AK, Moslehian, MS: Fuzzy almost quadratic functions. Results Math. 52, 161-177 (2008). doi:10.1007/ s00025-007-0278-9

24. Jung, S-M: On the Hyers-Ulam stability of the functional equations that have the quadratic property. J Math Anal Appl. 222, 126-137 (1998). doi:10.1006/jmaa.1998.5916

doi:10.1186/1029-242X-2011-70

Cite this article as: Jin and Lee: Fuzzy stability of a mixed type functional equation. Journal of Inequalities and Applications $20112011: 70$.

\section{Submit your manuscript to a SpringerOpen ${ }^{\circ}$} journal and benefit from:

- Convenient online submission

- Rigorous peer review

- Immediate publication on acceptance

- Open access: articles freely available online

- High visibility within the field

- Retaining the copyright to your article

Submit your next manuscript at $\boldsymbol{s p r i n g e r o p e n . c o m ~}$ 